\title{
Can Rituximab Improve the Outcome of Patients with Nodular Lymphocyte- Predominant Hodgkin's Lymphoma?
}

\author{
Heidi Mocikova ${ }^{a}$ Robert Pytlik ${ }^{b}$ Pavla Stepankovac Jozef Michalka ${ }^{d}$ \\ Jana Markova $^{a}$ Jan Koren ${ }^{b}$ Lucie Buresova ${ }^{\mathrm{e}}$ Ludek Raida ${ }^{f}$ Zdenek Kral $^{d}$ \\ a Internal Clinic of Hematology, University Hospital Královské Vinohrady, Third Faculty of Medicine, Charles \\ University in Prague, ${ }^{b}$ First Medical Department - Clinical Department of Hematooncology, First Faculty of \\ Medicine and General Teaching Hospital, Charles University in Prague, Prague, ' Fourth Department of Internal \\ Medicine - Hematology, Faculty of Medicine and University Hospital, Charles University, Hradec Králové, \\ ${ }^{d}$ Department of Internal Medicine - Hematology and Oncology, University Hospital Brno, e DSC Services, s.r.o., \\ Tisnov, ${ }^{\mathrm{f}}$ Department of Hemato-Oncology, Faculty of Medicine and Dentistry, Palacky University, \\ Olomouc, Czech Republic
}

For editorial comment see p. 185

\section{Key Words}

Chemotherapy · Hodgkin's lymphoma · Nodular

lymphocyte-predominant Hodgkin's lymphoma · Rituximab treatment. Conclusion: The prognosis of NLPHL is excellent. Rituximab combined with chemotherapy and/or radiotherapy appears to prevent disease progression/relapse.

(c) 2015 S. Karger AG, Base

\begin{abstract}
Background: Nodular lymphocyte-predominant Hodgkin's lymphoma (NLPHL) is a rare subtype of Hodgkin's lymphoma showing strong CD20 expression. The role of rituximab in treating NLPHL still needs clarification. Methods: We retrospectively reviewed the outcome of 23 patients with NLPHL treated with rituximab alone or in combination with chemotherapy and/or radiotherapy as part of their first- or secondline treatment. Results: The median follow-up of the whole group was 67 months, and all patients remained alive. Twenty-two patients achieved complete remission after rituximab-based therapy, and one of them relapsed 32 months after treatment. One patient treated with rituximab alone achieved partial remission and progressed 22 months after
\end{abstract}

\section{Introduction}

Nodular lymphocyte-predominant Hodgkin's lymphoma (NLPHL) accounts for 5\% of all Hodgkin's lymphomas (HLs) and has a better prognosis than classical HL [1]. The course of NLPHL is indolent, with delayed relapses. A large retrospective analysis based on the German Hodgkin Study Group (GHSG) database demonstrated that at a median observation time of 50 months the freedom from treatment failure rates were $93 \%$ for early, $87 \%$ for intermediate and $77 \%$ for advanced stages of NLPHL [1]. Patients with NLPHL in clinical stage IA without risk factors have an extremely favorable progno-

\section{KARGER 125\%}

() 2015 S. Karger AG, Base

0001-5792/15/1343-0187\$39.50/0

E-Mail karger@karger.com

www.karger.com/aha
Heidi Mocikova, MD, PhD

Internal Clinic of Hematology, University Hospital Královské Vinohrady

Third Faculty of Medicine, Charles University in Prague

Srobarova 50, CZ-100 34 Prague 10 (Czech Republic)

E-Mail heidi.mocikova@ seznam.cz 
sis, with excellent long-term outcome, and they should receive involved-field radiotherapy (IFRT) of $30 \mathrm{~Gy}$ as frontline treatment $[2,3]$. According to the guidelines proposed by the European Society for Medical Oncology, all patients with NLPHL other than those with clinical stage IA without risk factors should be treated identically to classical HL, i.e. with systemic chemotherapy and/or radiotherapy [4].

A retrospective review of 37 patients with NLPHL from Cancer and Leukemia Group B trials (8251 and 8952) suggested that MOPP, a classical alkylating agentcontaining regimen (i.e. mechlorethamine, vincristine, procarbazine and prednisone), led to fewer treatment failures compared with ABVD (doxorubicin, bleomycin, vinblastine and dacarbazine) [5]. Fanale et al. [6] retrospectively analyzed the outcome of 63 patients with NLPHL who received R-CHOP or other regimens. The overall response rate was $100 \%$, with $90 \%$ complete remissions (CR) being observed. None of the R-CHOP patients relapsed within a median follow-up of 42 months (range 8-111 months). However, 19\% of patients treated with other therapies relapsed after a median follow-up of 38 months (range 4-72 months). Compared with other treatments, R-CHOP shows a trend towards improved progression-free survival (PFS) at 5 years (95\% for R$\mathrm{CHOP}$ and $71 \%$ for other therapies). Xing et al. [7] conducted a retrospective matched-pair outcome analysis of 42 patients with advanced-stage NLPHL paired 1:2 with classical HL. The majority of patients received ABVDlike chemotherapy. The 10-year overall survival (OS) and freedom from treatment failure were similar in NLPHL and classical HL (75 vs. $73 \%, p=0.610$ ), but 10 -year time to progression was inferior in NLPHL (63 vs. $73 \%, \mathrm{p}=$ $0.040)$. Splenic involvement was associated with an inferior 10-year time to progression in patients treated with ABVD (48 vs. $71 \%, p=0.049$ ) and with an increased cumulative incidence of transformed aggressive lymphomas $(p=0.014)$. Other studies have also reported an increased risk of transformation to aggressive B-cell nonHL in NLPHL compared with classical HL, which is seen mainly in patients with advanced stages of NLPHL and splenic involvement at diagnosis [8]. However, most patients $(79 \%)$ who exhibited transformation into aggressive lymphoma had been diagnosed with NLPHL stage I/ II in the study by Biasoli et al. [9].

Strong CD20 expression and low acute toxicity suggest the feasibility of using rituximab in the treatment of NLPHL. A new prognostic score for relapse/progression was recently proposed by the GHSG based on the histopathological subtype (typical vs. variant NLPHL), serum albumin ( $\geq 4$ vs. $<4 \mathrm{~g} / \mathrm{dl}$ ) and gender (female vs. male) [10]. This prognostic score identifies patients at a higher risk of treatment failure when they are treated with current standard approaches. The authors suggested that these patients may be candidates for novel treatment strategies, including the use of targeted drugs such as CD20 antibodies. However, rituximab is inferior to radiotherapy alone in the first-line treatment of patients with NLPHL in clinical stage IA: the reported 3-year PFS rate is $81.4 \%$ after rituximab alone [11], and the 5-year PFS rate is $95 \%$ after radiotherapy [3]. The Stanford Group applied rituximab induction with or without maintenance in 39 patients with untreated and relapsed NLPHL. Despite the $100 \%$ overall response rate, the estimated 5 -year PFS and OS for patients treated with rituximab versus rituximab combined with maintenance therapy were only 39.1 and 95.7 versus 58.9 and $85.7 \%$, respectively. Rituximab did not prevent transformation into aggressive B-cell non-HL in 9 of 23 relapsing patients [12].

The aim of this analysis was to assess the role of rituximab as first- or second-line treatment, either alone or in combination with other treatment modalities.

\section{Patients and Methods}

\section{Patients}

This study summarizes the outcome of 23 NLPHL patients treated with rituximab (with or without chemotherapy/radiotherapy) in five centers from the Czech Republic between 2006 and 2014. The diagnosis of NLPHL was reviewed by specialized hematopathologists. Fan et al. [13] described two typical histopathological growth patterns in NLPHL with predominantly nodular growth: B-cell-rich nodular (pattern A) and serpiginous/interconnected growth (pattern B). They also characterized the following histopathological variants: extranodular lymphocyte-predominant cells (pattern C), a T-cell-rich (B-cell-depleted) nodular pattern (pattern D), a diffuse T-cell/histiocyte-rich large B-cell lymphoma-like pattern (pattern E) and a diffuse moth-eaten pattern (pattern F) [13]. Variant NLPHL histologies (non-A/B pattern) were identified in 5 of 23 patients. Based on the prognostic score proposed by Hartmann et al. [10], patients were divided into three risk groups: a low-risk group $(\mathrm{n}=5)$, an intermediate-risk group $(n=11)$ and a high-risk group $(n=7)$. The characteristics of the patients are summarized in table 1 . The median age of all patients at diagnosis was 33.2 (16-65) years. The median age at the onset of rituximab treatment is recorded in table 1. Risk factors at the diagnosis of NLPHL were evaluated according to the risk stratification applied in classical HL. Of the 23 NLPHL patients, 13 received frontline rituximab, and 10 were treated with second-line rituximab therapy (table 2).

Rituximab as First-Line Treatment

Among the 7 patients in early clinical stage I or II, rituximab alone $\left(375 \mathrm{mg} / \mathrm{m}^{2}\right.$ i.v. weekly $\left.\times 4\right)$ was given to 2 patients. Ritux- 
Table 1. Characteristics of the NLPHL patients $(n=23)$

\begin{tabular}{lll}
\hline Characteristics & $\begin{array}{l}\text { First-line } \\
\text { rituximab } \\
(\mathrm{n}=13)\end{array}$ & $\begin{array}{l}\text { Second-line } \\
\text { rituximab } \\
(\mathrm{n}=10)\end{array}$ \\
\hline $\begin{array}{l}\text { Median age at rituximab treatment, } \\
\quad \text { years (range) }\end{array}$ & $38(20-65)$ & $34(16-44)$ \\
$\begin{array}{l}\text { Gender (males/females) } \\
\text { Clinical stage }\end{array}$ & $11 / 2$ & $7 / 3$ \\
$\quad$ I & 3 & 1 \\
II & 4 & 5 \\
$\quad$ III & 5 & 3 \\
$\quad$ IV & 1 & 1 \\
B symptoms present & 2 & 2 \\
Stage IA without risk factors & 3 & - \\
Risk factors present & 4 & - \\
\hline
\end{tabular}

Risk factors are a large mediastinal mass ( $\geq 1 / 3$ of the maximum transverse diameter of the thorax); extranodal disease; a high erythrocyte sedimentation rate $\geq 50$ without $B$ symptoms and $\geq 30$ with B symptoms present, and involvement of $\geq 3$ lymph node areas.

imab with IFRT (30 Gy) was administered to 2 patients [ 2 cycles of R-ABVD + IFRT of 30 Gy in 2 patients and 4 cycles of escalated R-BEACOPP (rituximab, bleomycin, etoposide, doxorubicin, cyclophosphamide, vincristine, prednisone and procarbazine) + IFRT of 30 Gy in 1 patient with clinical stage IIB and risk factors].

Among the 6 patients in advanced stage III or IV, 1 received rituximab alone; 2 patients were treated with 6 cycles of R-ABVD, and 3 patients received 6 cycles of R-CHOP.

\section{Rituximab as Second-Line Treatment}

None of the 10 relapsing patients received frontline rituximab, and their first-line treatment included a combination of ABVD and IFRT (7 patients), COPP (cyclophosphamide, vincristine, procarbazine and prednisone) and IFRT (1 patient) or IFRT alone (3 patients).

Six of these patients were in clinical stage I or II at the time of the first relapse: 3 received rituximab alone; 2 patients were treated with 6 cycles of R-ABVD, and 1 patient was administered 2 cycles of escalated R-BEACOPP + 2 cycles of R-ABVD + IFRT of $30 \mathrm{~Gy}$. Four of the relapsing patients were in an advanced clinical stage: 3 patients received 6 cycles of R-BEACOPP and 1 patient (pretreated with ABVD) was treated with 3 cycles of R-DHAP (rituximab, dexamethasone, cytarabine and cisplatin) and subsequently underwent high-dose chemotherapy and autologous stem cell transplantation (ASCT).

\section{Statistics}

PFS was calculated from the date of the initiation of rituximab treatment until the next relapse. OS was calculated from the date of diagnosis until lymphoma-related death or death from any other reason. Survival curves were calculated using Kaplan-Meier sur-
Table 2. Treatment of patients with rituximab (R) as first- or second-line treatment $(n=23$ patients)

\begin{tabular}{|c|c|c|}
\hline Treatment & $\begin{array}{l}\text { First } \\
\text { line } \\
(n=13)\end{array}$ & $\begin{array}{l}\text { Second } \\
\text { line } \\
(n=10)\end{array}$ \\
\hline $4 \times \mathrm{R}$ alone & 3 & 3 \\
\hline \multicolumn{3}{|l|}{$\mathrm{R}+$ chemotherapy } \\
\hline $6 \times \mathrm{R}-\mathrm{ABVD}$ & 2 & 2 \\
\hline $6 \times \mathrm{R}-\mathrm{BEACOPP}$ & 0 & 3 \\
\hline $6 \times \mathrm{R}-\mathrm{C}(\mathrm{H}) \mathrm{OP}$ & 3 & 0 \\
\hline $3 \times \mathrm{R}-\mathrm{DHAP}$ & 0 & 1 \\
\hline $4 \times \mathrm{R}+\mathrm{RT}$ & 2 & 0 \\
\hline \multicolumn{3}{|l|}{$\mathrm{R}+$ chemotherapy $+\mathrm{RT}$} \\
\hline $4 \times \mathrm{R}-\mathrm{BEACOPP}+\mathrm{RT}$ & 1 & 0 \\
\hline $2 \times \mathrm{R}-\mathrm{BEACOPP}+2 \times \mathrm{R}-\mathrm{ABVD}+\mathrm{RT}$ & 0 & 1 \\
\hline $2 \times \mathrm{R}-\mathrm{ABVD}+30$ Gy RT & 2 & 0 \\
\hline \multicolumn{3}{|l|}{ Treatment response } \\
\hline CR & 13 & 9 \\
\hline PR & 0 & 1 \\
\hline
\end{tabular}

$\mathrm{RT}=$ Radiotherapy $\mathrm{PR}=$ partial remission . vival analysis, and comparisons between subgroups were performed using the log-rank test. STATISTICA (StatSoft Inc., Tulsa, Okla., USA) and R software (R Core Team, 2012) were employed for statistical analysis.

\section{Results}

The median follow-up of the whole group lasted 67 (range 3-257) months: 57 (range 3-88) months for firstline rituximab, 112 (49-257) months for second-line rituximab since diagnosis and 49 (range 16-61) months since the first relapse. All of the patients treated with rituximab \pm chemotherapy/radiotherapy as first-line treatment achieved CR, and none of them relapsed.

Among the 10 patients treated with rituximab \pm chemotherapy and/or radiotherapy as second-line treatment, 9 achieved CR, and 1 patient treated with rituximab alone achieved partial remission. The patient in partial remission received no further treatment and progressed after 22 months. Another patient in clinical stage II treated with rituximab alone as second-line treatment achieved a second CR and relapsed after 32 months. Both relapsing patients treated with second-line rituximab were in the intermediate-risk group at their initial diagnosis according to Hartmann et al. [10]. Despite the higher rate of hematological toxicities and infections ob- 


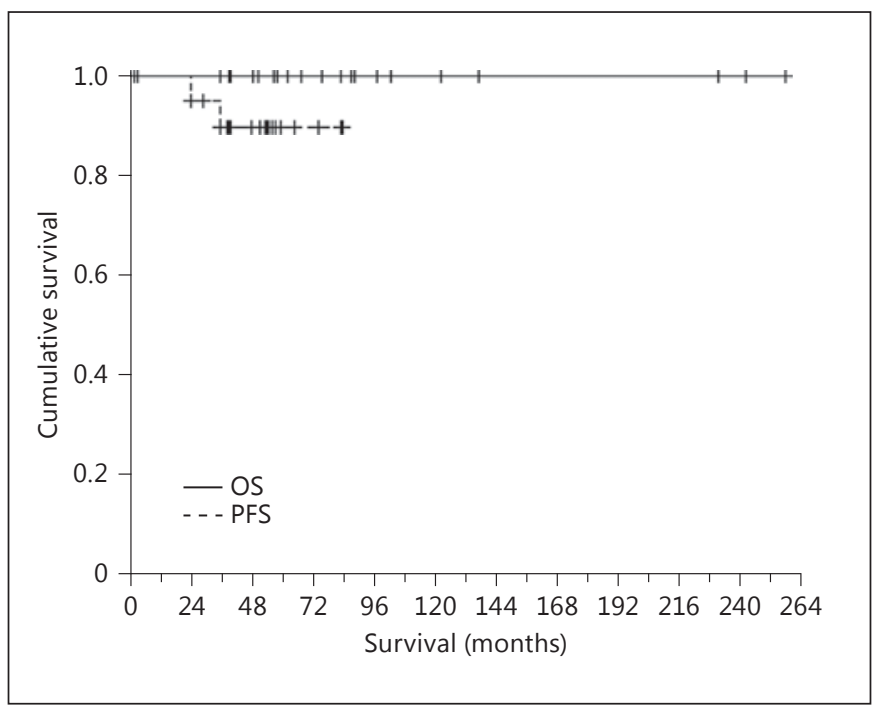

Fig. 1. PFS and OS in NLPHL patients $(n=23)$.

served in patients treated with aggressive approaches such as R-BEACOPP or high-dose chemotherapy and ASCT, these therapies did not lead to death or permanent serious organ damage.

None of all these patients died during the follow-up, and the 5 -year OS of the whole group was $100 \%$ (fig. 1). The 2- and 5-year PFS in the whole group were 95\% [95\% confidence interval (CI), 0.87-1.00] and 90\% (95\% CI, $0.78-1.00$ ), respectively (fig. 1). The 2- and 5-year PFS in patients treated with rituximab as first-line treatment were both $100 \%$ (fig. 2). The 2 - and 5 -year PFS in patients treated with rituximab as second-line treatment were $89 \%$ (95\% CI, 0.71-1.00) and 76\% (95\% CI, 0.52-1.00), respectively (fig. 2). PFS in patients treated with rituximab as first-line treatment did not differ significantly from PFS in patients treated with rituximab as secondline treatment $(\mathrm{p}=0.089)$.

Histological transformation to an aggressive type of lymphoma was not observed during the median followup of 67 months. No serious long-term toxicity was reported in this group of patients.

\section{Discussion}

There is little evidence regarding the optimal treatment for NLPHL due to the rarity of this disease. The favorable outcome of stage I or II patients without risk factors was mainly determined on the basis of retrospective

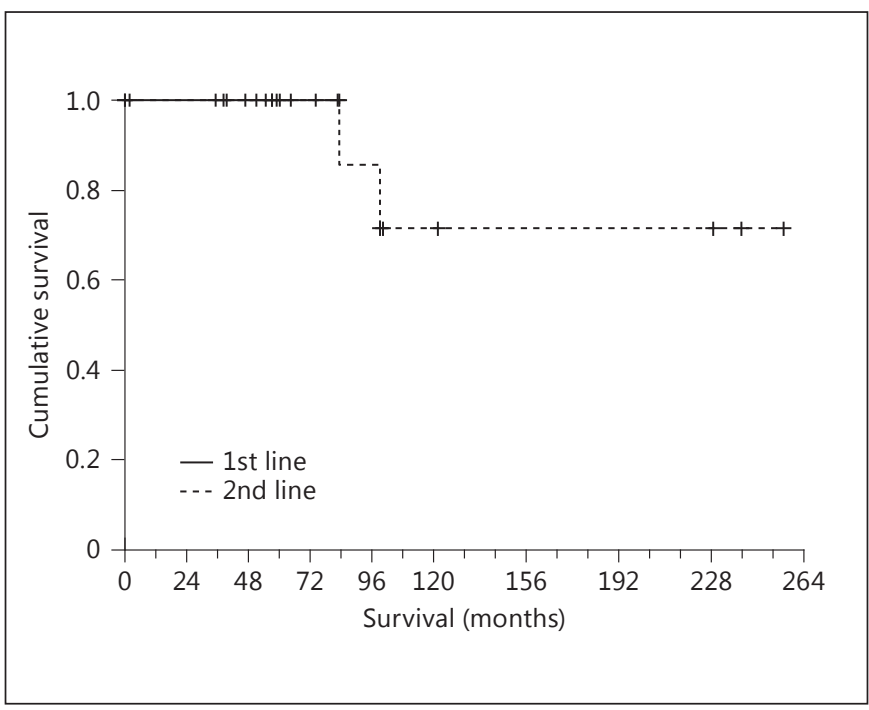

Fig. 2. Rituximab as first- $(\mathrm{n}=13)$ and second-line $(\mathrm{n}=10)$ treatment and PFS.

studies, from which localized radiotherapy (involved field) emerged as an optimal initial approach in patients showing stage IA without risk factors. PFS for stage II patients receiving IFRT alone is worse than for stage I patients, and it is unclear whether this is an optimal approach for such patients. There is even less evidence regarding the treatment of advanced or relapsed disease, where approaches for advanced classical HL are frequently used.

Rituximab is effective in the first- or second-line setting in NLPHL and is attractive due to its low toxicity. However, the optimal use of rituximab must be determined. From our small, heterogeneous group, it is difficult to draw definite conclusions, but several potentially important observations were made.

Rituximab was used in 7 previously untreated patients in early clinical stages, and none of these patients relapsed during the median follow-up of 57 months. This group included 2 patients treated with rituximab alone, 2 patients in whom radiotherapy was added and 3 patients treated with a combination of rituximab, chemotherapy and radiotherapy. Although the follow-up was relatively short for this disease which is characterized by late relapses, the idea that rituximab added to radiotherapy and/or short chemotherapy might reduce the risk of relapse - while adding no extra toxicity - appears to be attractive.

Potentially more interesting are our observations made in patients with intermediate and advanced disease 
and during second-line treatment. For patients with stage II disease, especially those with risk factors, the relapse rate is higher than for stage I disease, while for patients with advanced-stage disease, the rate of relapse is similar to that of classical HL. However, none of 4 previously untreated patients showing clinical stage II and 6 patients with advanced disease relapsed after a median follow-up of 57 months. Only 1 of these patients received monotherapy with rituximab, while all the others received combined treatment. Based on Stanford results obtained in advanced stages, including both newly diagnosed and relapsed patients, the observed responses using rituximab alone were not durable (median PFS, 22 months) [12], and our results therefore appear to be better.

A temporary effect of rituximab alone was confirmed in a GHSG trial in relapsed NLPHL patients [14], showing a high response rate with a median time to progression of 33 months. However, none of 7 patients treated with rituximab and varieties of different chemotherapy/ radiotherapy regimens relapsed during the median follow-up of 49 months. In the relapsed setting, chemotherapy with rituximab achieves a favorable outcome. It is sometimes not possible to apply combination therapy including rituximab and conventional chemotherapy protocols such as CHOP, ABVD or BEACOPP in relapsed patients depending on the type of frontline treatment, e.g. due to the cumulative anthracycline dose, and an alternative treatment, such as R-DHAP, may be used, especially in advanced stages. One relapsed patient in clinical stage
IV underwent ASCT in their second complete remission. Only a small number of relapsed NLPHL patients underwent high-dose chemotherapy and ASCT $[9,15,16]$, and it is difficult to assess the role ASCT in this setting. Moreover, ASCT does not prevent further relapses: Jackson et al. [15] analyzed 8 patients treated with ASCT, 5 of whom experienced relapse after ASCT and received subsequent chemotherapy.

Based on our data and those from other studies, we propose the following approach for treating NLPHL: as first-line treatment, in stage IA patients without risk factors, radiotherapy alone is sufficient, whereas all patients other than those with stage IA without risk factors should be treated with rituximab and chemotherapy. The prognosis of NLPHL is excellent in terms of OS. However, rituximab alone is not sufficient to prevent relapses after first- or second-line treatment. The suggested treatment approach is not based on prospective data, and further prospective studies are needed to define the optimal chemoimmunotherapy regimen with the most favorable efficacy/toxicity profile in NLPHL as frontline treatment and following relapse.

\section{Acknowledgement}

The study was supported by Research Project P 27/2012 (Third Faculty of Medicine, Charles University in Prague, Prague, Czech Republic).

\section{References}

1 Nogová L, Reineke T, Brillant C, Sieniawski $\mathrm{M}$, Rüdiger $\mathrm{T}$, Josting $\mathrm{A}$, Bredenfeld $\mathrm{H}$, Skripnitchenko R, Müller RP, Müller-Hermelink HK, Diehl V, Engert A; German Hodgkin Study Group: Lymphocyte-predominant and classical Hodgkin's lymphoma: a comprehensive analysis from the German Hodgkin Study Group. J Clin Oncol 2008;26:434-439.

-2 Nogová L, Reineke T, Eich HT, Josting A, Müller-Hermelink HK, Wingbermühle $\mathrm{K}$, Brillant C, Gossmann A, Oertel J, Bollen MV, Müller RP, Diehl V, Engert A: Extended field radiotherapy, combined modality treatment or involved field radiotherapy for patients with stage IA lymphocyte-predominant Hodgkin's lymphoma: a retrospective analysis from the German Hodgkin Study Group (GHSG). Ann Oncol 2005;16:1683-1687.

-3 Chen RC, Chin MS, Ng AK, Feng Y, Neuberg D, Silver B, Pinkus GS, Stevenson MA, Mauch PM: Early-stage, lymphocyte-predominant Hodgkin's lymphoma: patient outcomes from a large, single-institution series with long follow-up. J Clin Oncol 2010;28:136-141.

-4 Eichenauer DA, Engert A, André M, Federico $\mathrm{M}$, Illidge T, Hutchings M, Ladetto M; ESMO Guidelines Working Group: Hodgkin's lymphoma: ESMO Clinical Practice Guidelines for diagnosis, treatment and follow-up. Ann Oncol 2014;25(suppl 3):iii70-iii75.

5 Canellos GP, Mauch P: What is the appropriate systemic chemotherapy for lymphocytepredominant Hodgkin's lymphoma? J Clin Oncol 2010;28:e8.

6 Fanale MA, Lai CM, McLaughlin P, Romaguera J, Fayad L, Hagemeister F, Samaniego F, Rodriguez MA, Neelapu SS, Shah JJ, Kwak L, Dong W, Reed V, Dabaja BS, Popat U, Younes A: Outcomes of nodular lymphocyte-predominant Hodgkin's lymphoma (NLPHL) patients treated with R-CHOP (abstract). Blood (ASH Annual Meeting Abstracts) 2010;116:2812.

-7 Xing KH, Connors JM, Lai A, Al-Mansour M, Sehn LH, Villa D, Klasa R, Shenkier T, Gascoyne RD, Skinnider B, Savage KJ: Advanced stage nodular lymphocyte predominant Hodgkin lymphoma compared to classical Hodgkin lymphoma: a matched pair outcome analysis. Blood 2014;123:3567-3573.

-8 Al-Mansour M, Connors JM, Gascoyne RD, Skinnider B, Savage KJ: Transformation to aggressive lymphoma in nodular lymphocyte-predominant Hodgkin's lymphoma. J Clin Oncol 2010;28:793-799.

-9 Biasoli I, Stamatoullas A, Meignin V, Delmer A, Reman O, Morschhauser F, Coiffier B, Bosly A, Diviné M, Brice P: Nodular, lymphocyte-predominant Hodgkin lymphoma. Cancer 2010;116:631-639.

10 Hartmann S, Eichenauer DA, Plütschow A, Mottok A, Bob R, Koch K, Bernd HW, Cogliatti S, Hummel M, Feller AC, Ott G, Möller P, Rosenwald A, Stein H, Hansmann ML, Engert A, Klapper W: The prognostic impact of variant histology in nodular lymphocyte-predominant Hodgkin lymphoma: a report from the German Hodgkin Study Group (GHSG). Blood 2013;122:4246-4252.
Rituximab and Outcome in NLPHL Patients 
11 Eichenauer DA, Fuchs M, Pluetschow A, Klimm B, Halbsguth T, Böll B, von Tresckow B, Nogová L, Borchmann P, Engert A: Phase 2 study of rituximab in newly diagnosed stage IA nodular lymphocyte-predominant Hodgkin lymphoma: a report from the German Hodgkin Study Group. Blood 2011;118:43634365.

12 Advani RH, Horning SJ, Hoppe RT, Daadi S, Allen J, Natkunam Y, Bartlett NL: Mature results of a phase II study of rituximab therapy for nodular lymphocyte-predominant Hodgkin lymphoma. J Clin Oncol 2014;32:912918.
13 Fan Z, Natkunam Y, Bair E, Tibshirani R, Warnke RA: Characterization of variant patterns of nodular lymphocyte predominant Hodgkin lymphoma with immunohistologic and clinical correlation. Am J Surg Pathol 2003;27:1346-1356.

14 Schulz H, Rehwald U, Morschhauser F, Elter $T$, Driessen C, Rüdiger T, Borchmann P, Schnell R, Diehl V, Engert A, Reiser M: Rituximab in relapsed lymphocyte-predominant Hodgkin lymphoma: long-term results of a phase 2 trial by the German Hodgkin Lymphoma Study Group (GHSG). Blood 2008; 111:109-111.
5 Jackson C, Sirohi B, Cunningham D, Horwich A, Thomas K, Wotherspoon A: Lymphocytepredominant Hodgkin lymphoma - clinical features and treatment outcomes from a 30year experience. Ann Oncol 2010;21:20612068.

16 Karuturi M, Hosing C, Fanale M, Medeiros LJ, Alousi AM, de Lima MJ, Qazilbash MH, Kebriaei P, Younes A, Khouri I, Andersson BS, Champlin R, Anderlini P, Popat U: High-dose chemotherapy and autologous stem cell transplantation for nodular lymphocyte-predominant Hodgkin lymphoma. Biol Blood Marrow Transplant 2013;19: 991-994. 